TecnoLógicas

ISSN-p 0123-7799

ISSN-e 2256-5337

Vol. 21, No. 42, pp. 129-145

Mayo-agosto de 2018

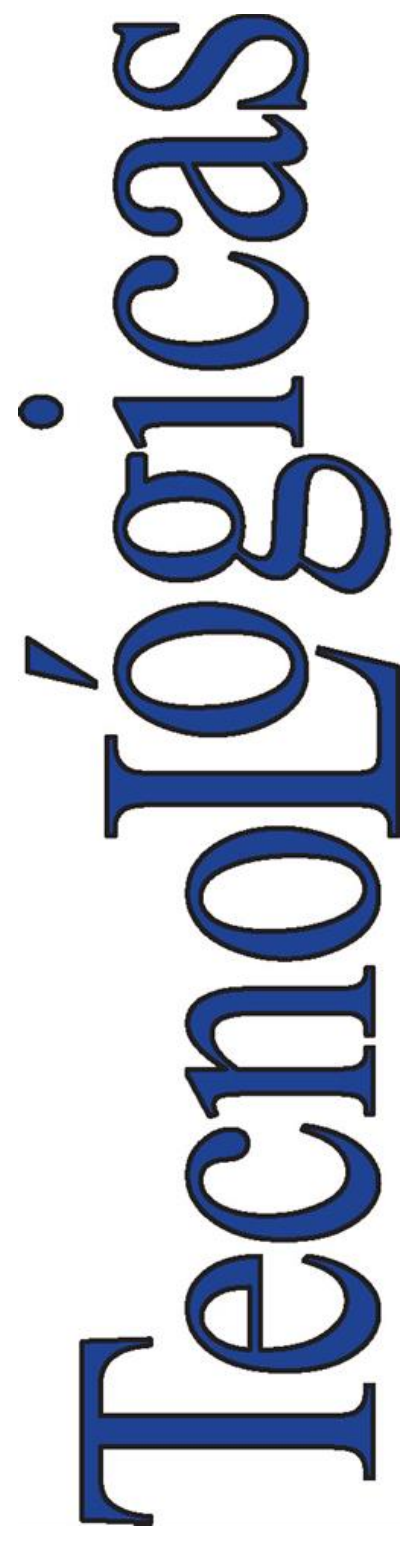

(C) Copyright 2015 por autores y Tecno Lógicas Este trabajo está licenciado bajo una Licencia Internacional Creative Commons Atribución (CC BY)

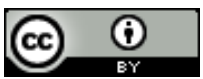

Artículo de Investigación/Research Article

\section{Cargador de baterías fotovoltaico con control por modos deslizantes y limitación de la derivada de corriente de carga}

\section{Photovoltaic battery charger with sliding mode control and charging current derivative limitation}

\author{
Carlos A. Ramos-Paja ${ }^{1}$, Andrés J. Saavedra-Montes ${ }^{2}$ \\ y Juan D. Bastidas-Rodríguez ${ }^{3}$
}

Recibido: 03 de noviembre de 2017

Aceptado: 15 de marzo de 2018

Cómo citar / How to cite

C. A. Ramos-Paja, A. J. Saavedra-Montes, y J. D. Bastidas-Rodríguez, Cargador de baterías fotovoltaico con control por modos deslizantes y limitación de la derivada de corriente de carga. TecnoLógicas, vol. 21, no. 42, pp. 129-145, 2018.

1 PhD en Ingeniería Electrónica, Automática y Comunicaciones, Magíster en Ingeniería Énfasis en Automática, Magíster en Ingeniería Electrónica Ingeniero Electrónico, Departamento de Energía Eléctrica y Automática, Universidad Nacional de Colombia, Medellín-Colombia, caramosp@unal.edu.co

$2 \mathrm{PhD}$ en Ingeniería, Magíster en Sistemas de Generación de Energía Eléctrica Ingeniero Electricista, Departamento de Energía Eléctrica y Automática, Universidad Nacional de Colombia, Medellín-Colombia, ajsaaved@unal.edu.co

$3 \mathrm{PhD}$ en Ingeniería, Ingeniero Electrónico, Escuela de Ingenierías Eléctrica, Electrónica y de Telecomunicaciones, Universidad Industrial de Santander, Bucaramanga-Colombia,jdbastir@uis.edu.co 



\title{
Resumen
}

En los sistemas fotovoltaicos (PV) aislados, los cargadores de baterías son importantes para garantizar el suministro de energía cuando la luz solar no está disponible. Dichos cargadores necesitan realizar el seguimiento del punto de máxima potencia (MPPT) y limitar la derivada de corriente de carga de las baterías para extender su vida útil. Este artículo propone un sistema cargador de baterías compuesto por un convertidor Buck, un control en cascada de la corriente de las baterías y la tensión del panel PV, y la técnica de MPPT Perturbar y Observar (P\&O). El P\&O genera la referencia de tensión del panel para el lazo externo del control en cascada, implementado con un regulador $\mathrm{P}$, cuya acción de control es la referencia de la corriente de carga de las baterías. Dicha referencia de corriente pasa por un limitador de derivada antes de llegar al lazo interno de control de corriente de las baterías, el cual es implementado con un control por modos deslizantes (SMC). El artículo incluye el análisis de transversalidad y alcanzabilidad del SMC, así como el procedimiento de diseño del regulador P. El sistema propuesto se valida por medio de simulaciones en el software PSIM mostrando la capacidad de realizar el MPPT y limitar la derivada de corriente de carga de las baterías al mismo tiempo.

\section{Palabras clave}

Cargador baterías, panel fotovoltaico, convertidor Buck, control por modos deslizantes, limitación derivada de corriente.

\begin{abstract}
In stand-alone photovoltaic (PV) systems, battery chargers are important to guarantee the energy supply when sunlight is not available. Such chargers need to track the maximum power point (MPPT) and limit the derivative of the batteries' charging current to extend their lifetime. This paper proposes a battery charging system composed of a Buck converter, a cascade control of the battery current and the PV panel voltage, and the Perturb and Observe (P\&O) MPPT technique. P\&O generates the reference of the panel voltage for the external loop of the cascade control implemented with a $\mathrm{P}$ regulator, whose control action is the reference of the batteries' charging current. Such current reference passes through a derivative limiter before reaching the internal current control loop, which is implemented by a sliding-mode controller (SMC). This paper includes transversality and reachability analyses of the SMC, as well as the procedure to design the $\mathrm{P}$ regulator. The proposed system is validated by simulations in PSIM software to show its capacity to perform MPPT and limit the battery's charging current derivative at the same time.
\end{abstract}

\section{Keywords}

Battery charger, photovoltaic panel, Buck converter, sliding-mode control, current derivative limitation. 


\section{INTRODUCCIÓN}

La necesidad de reducir los gases de efecto invernadero asociados a la producción de energía y de contribuir con el desarrollo sostenible global ha provocado que, en los últimos cinco años, la inversión en generación con fuentes renovables haya sido mayor a la inversión en generación con combustibles fósiles [1]. Solamente en 2016 se estima que la potencia instalada en energías renovables fue de $161 \mathrm{GW}$, de los cuales el $47 \%$ corresponde a generación fotovoltaica (PV por sus siglas en inglés), ubicándola como la fuente renovable de mayor crecimiento en 2016 [1], [2].

Colombia cuenta con un potencial significativo para la generación de energía PV [3], [4] que puede ser explotado tanto en las zonas conectadas al sistema de interconexión nacional como en las Zonas No Interconectadas (ZNI). Sin embargo, la implementación de sistemas de generación PV en ZNI podría tener un mayor impacto en la calidad de vida de los habitantes con la implementación de diferentes aplicaciones de bajo costo: puntos de carga de dispositivos electrónicos, sistemas de iluminación en hogares, aplicaciones de alumbrado público, alimentación de sistemas de comunicaciones, entre otras [5].

Los sistemas PV no conectados a la red (o aislados) requieren de un sistema de almacenamiento de energía (típicamente baterías) para poder alimentar las cargas durante la noche o cuando la irradiación solar no es suficiente [6], [7]. Por lo tanto, la energía producida por los generadores PV se debe utilizar para cargar las baterías, que es el punto de donde se alimenta la carga.

Un cargador de baterías alimentado por un generador PV está compuesto de cuatro partes principales: el generador PV, un convertidor de potencia, un banco de baterías y un sistema de control [6], [7]. La tensión del generador PV usualmente es mayor a la tensión del banco de baterías, por lo tanto se necesita un convertidor reductor para acoplar el generador PV al banco de baterías [8]. El sistema de control se encarga de extraer la máxima potencia del generador PV, modificando su tensión, y de regular la corriente entregada a la batería para mantenerla dentro de los límites requeridos. Adicionalmente, el sistema de control puede incluir límites máximos para la corriente de carga de las baterías y su derivada [9], [10]. Estos límites ayudan a extender la vida útil de las baterías, ya que a mayores valores de las corrientes de carga [11] y su derivada, menor será la vida útil de las baterías [9], [10], [12], [13].

Uno de los indicadores más importantes de la vida útil de las baterías es el número de ciclos completos de carga/descarga antes de fallar (CTF por sus siglas en inglés) [9], [14]. Dicho número depende, principalmente, de la profundidad de descarga y de la magnitud de la corriente de carga/descarga, de acuerdo con el modelo propuesto en [10]. La profundidad de descarga afecta directamente el CTF en concordancia a lo expresado en [9], [14], pues en una batería la profundidad de descarga es proporcional a la sustancia activa transformada en cada ciclo; por lo tanto, a mayor profundidad de descarga, mayor consumo de la vida útil de la batería. Por otro lado, la magnitud de las corrientes de carga/descarga reducen la capacidad de almacenamiento de energía en las baterías como muestran los experimentos realizados en [11]. Los resultados muestran que, a mayor magnitud de corriente, mayor reducción en la capacidad de almacenamiento de las baterías y, como consecuencia, menor vida útil.

Típicamente, un ciclo de carga/descarga en una batería se contabiliza cuando la batería llega hasta su mínimo valor de carga (recomendado por el fabricante) y vuelve a cargarse completamente. Sin embargo, los ciclos de carga/descarga no siempre se producen de forma completa, por el comportamiento de la carga alimentada por las baterías y/o por el sistema de 
carga de las mismas, lo que conlleva a ciclos incompletos de carga/descarga o subciclos [10]. Cuando el proceso de carga/descarga presenta corrientes con componentes de alta frecuencia y/o derivadas relativamente altas, se producen pequeños sub-ciclos de carga/descarga. Dichos subciclos se pueden sumar para contabilizar ciclos completos de carga/descarga que se tienen en cuenta para estimar la vida útil remanente en la batería. Por lo tanto, limitando las derivadas de corriente en los procesos de carga/descarga de las baterías se puede incrementar la vida útil de las mismas de forma significativa [9], [10], [12], [13]. Es por eso que en diferentes artículos se proponen sistemas de almacenamiento de energía híbridos compuestos por baterías y un dispositivo de almacenamiento de carga/descarga rápida como supercapacitores [9] o sistemas de almacenamiento magnético con superconductores [10], [12], [13]. Los dispositivos de almacenamiento rápido suministran/absorben las corrientes de altas frecuencias para que las baterías suministren/absorban las corrientes de bajas frecuencias, esto con el fin de aumentar la vida útil de las baterías.

En la literatura se encuentran sistemas de cargadores de baterías con generadores PV que utilizan diferentes convertidores de potencia y diferentes sistemas de control. En algunos artículos se utilizan convertidores reductores [8], [15], en otros utilizan convertidores elevadores [5] y en algunos casos convertidores elevadores de gran ganancia [16]-[18]. La selección del tipo de convertidor depende de la relación entre los niveles de tensión del generador PV y del banco de baterías de cada aplicación.

En cuanto a los sistemas de control del arreglo PV, la estrategia más simple es regular la tensión del generador PV a un valor constante donde teóricamente se encuentra el punto de máxima potencia [5]. Sin embargo, dicha estrategia no aprovecha la máxima potencia que puede entregar el generador PV. Para extraer la máxima potencia del generador PV se requie- re de una estrategia de seguimiento del punto de máxima potencia (MPPT por sus siglas en inglés), la cual se encarga de buscar la tensión del generador PV en la que entrega la máxima potencia. Dicho valor de tensión se utiliza como referencia para un regulador de tensión que manipula el ciclo de trabajo del convertidor de potencia para llevar la tensión del generador PV al valor deseado.

En los últimos años se han propuesto técnicas de MPPT que utilizan controladores en modos deslizantes (SMC por sus siglas en inglés) en el lazo interno para regular una variable en el convertidor [19]-[22] y un lazo externo para realizar el seguimiento del punto de máxima potencia (MPP por sus siglas en inglés) como Perturbar y Observar (P\&O) o Control de Búsqueda en Extremos (ESC por sus siglas en inglés). En [19], [20] se utiliza el P\&O para realizar el seguimiento del punto de máxima potencia. Por un lado, en [19] el $\mathrm{P} \& O$ genera una tensión de referencia que debe ser seguida por un controlador lineal, cuya acción de control es la referencia de un SMC de la corriente del capacitor de entrada de un convertidor Boost conectado al generador PV. Por otro lado, el P\&O propuesto en [20] calcula una admitancia de referencia en la entrada del generador PV para realizar el seguimiento del MPP; dicha admitancia es impuesta por medio de un convertidor Boost y un SMC que utiliza las mediciones de tensión y de corriente de entrada al convertidor. Otro algoritmo utilizado para realizar el MPPT es el ESC como se muestra en [21], [22]. En dichas referencias se propone controlar un convertidor Boost por medio de un SMC para que opere como un resistor sin pérdidas (LFR por sus siglas en inglés) que está conectado en paralelo al generador PV. De esta forma el ESC calcula el valor del LFR para que el generador PV opere en el MPP.

Respecto a los procedimientos de carga de las baterías existen diferentes métodos que difieren en complejidad del hardware requerido y en las características eléctricas 
de operación [23]. Dichos métodos van desde los más sencillos, como inyectar una corriente constante a la batería o fijar una tensión constante en sus terminales, hasta los más complejos que están definidos en varias etapas, dependiendo de la composición química de la batería y de variables como la temperatura, la corriente, la tensión y el estado de carga [24]. También se pueden encontrar en la literatura procedimientos que aprovechan la ecuación de carga de la batería, o la regla del amperiohora, para definir la corriente de carga según la capacidad de la batería durante la carga [23]. Sin embargo, dos métodos utilizados comúnmente para cargar la baterías son: corriente constante - tensión constante (CC-CV) [23] y corriente constante - tensión constante - tensión constante (CC-CV$\mathrm{CV})$ [25]. El método $\mathrm{CC}-\mathrm{CV}$ realiza una carga inicia con una corriente constante entre el $10 \%$ y el $25 \%$ de la capacidad de la batería hasta que la tensión llega a un valor de referencia $\left(V_{\text {ref } 1}\right)$. Luego, el control de carga fija la tensión en $V_{\text {refi }}$ hasta que la corriente de carga se encuentra entre un $0,4 \%$ y $0,5 \%$ de la capacidad de la batería. Por su parte, el método CC-CV-CV adiciona una etapa al método de CC-CV cambiando la referencia de tensión a un nuevo valor de referencia ( $\left.V_{\text {ref } 2}\right)$, denominado voltaje flotante, cuando la corriente de carga llega a su valor mínimo (aproximadamente $0,2 \%$ de la capacidad de la batería). Es importante notar que los valores de $V_{\text {ref } 1}$ y $V_{\text {ref2 }}$ se definen por los fabricantes de las baterías y que $V_{\text {ref } 2}<V_{\text {ref } 1}$.

En la literatura también se encuentran referencias importantes de cargadores de baterías alimentados por generadores PV. Por ejemplo, el sistema cargador de baterías PV propuesto en [7] cuenta con un algoritmo de control que integra una técnica de MPPT y una técnica de gestión de carga de la batería. Por un lado, la técnica de MPPT está basada en un algoritmo genético (GA) que estima el punto de máxima potencia del panel a partir de las mediciones de corriente, tensión, tempera- tura e irradiancia del panel. Por otro lado, la técnica de carga de la batería inicia con corriente constante hasta que la tensión llega a un valor de referencia; después, se carga a voltaje constante. Sin embargo, los autores no explican cómo implementar los controladores de bajo nivel para regular la tensión y la corriente en la batería. Adicionalmente, el sistema de control propuesto no garantiza la estabilidad del sistema para las diferentes condiciones de operación.

En los cargadores de batería PV presentados en [17], [18] los autores utilizan un regulador tipo PI para la tensión del generador PV y dos opciones de convertidor, un convertidor Zeta aislado y un convertidor 2Zeta. No obstante, los controladores tipo PI no pueden garantizar la estabilidad del sistema en un rango amplio de condiciones de operación, pues los convertidores son sistemas no-lineales y los modelos utilizados para el diseño de los controladores son modelos de pequeña señal, es decir, son válidos en una zona de operación restringida del convertidor.

Una forma de mejorar el sistema de control de un cargador de baterías es implementar un control en cascada, como se propone en [8], donde el lazo interno es un control PI de corriente de la batería y el lazo externo es un control de tensión de la batería. En [8] los autores también proponen un algoritmo de gestión de carga de la batería teniendo en cuenta sus niveles máximos y mínimos de tensión y corriente. No obstante, el sistema de control lineal propuesto en [8] está basado en un modelo de pequeña señal del convertidor, por lo tanto, el sistema de control no garantiza la estabilidad del sistema en cualquier condición de operación del cargador.

Otros autores proponen SMCs para obtener sistemas cargadores de baterías PV más robustos [15], [26]. En [15] se propone un SMC para regular la tensión del generador PV utilizando un convertidor Buck. La tensión de referencia del SMC es calculada por medio de una ecuación explícita 
que entrega un valor aproximado de la tensión del generador PV en el MPP. Por su parte en [26] se propone un control en cascada donde el lazo interno es un SMC de la corriente del inductor de un convertidor Buck-Boost y el lazo externo es un regulador PI de la tensión en la baterías. Aunque los controladores propuestos en [15], [26] son más robustos que los controladores lineales, en ninguno de los dos artículos se presenta el análisis de transversalidad, alcanzabilidad y control equivalente para los SMC propuestos; adicionalmente, en ninguno de los dos artículos se implementa una estrategia de MPPT robusta ante condiciones variables de irradiación solar y de temperatura.

Este artículo propone un sistema cargador de baterías alimentado por un generador PV utilizando un convertidor Buck capaz de seguir el punto de máxima potencia y limitar la derivada de la corriente entregada a la batería. La estrategia de control es un sistema en cascada, donde el lazo interno es un SMC de la corriente entregada a la batería y el lazo externo es un regulador tipo $\mathrm{P}$ de la tensión del generador. La referencia del SMC, i.e. la corriente entregada a la batería, es generada por el regulador $\mathrm{P}$ y pasa por un limitador de derivada antes de llegar al SMC, esto con el fin de asegurar que la corriente de la batería cumpla con las restricciones dinámicas de seguridad. Finalmente, la referencia de tensión del generador PV es calculada utilizando la técnica de Perturbar y Observar (P\&O) para extraer la máxima potencia del generador $\mathrm{PV}$. El sistema propuesto es implementado en el software PSIM para su validación. Los resultados muestran que el sistema propuesto es capaz de realizar el seguimiento del punto de máxima potencia y limitar la derivada de la corriente entregada a la batería ante condiciones de irradiación solar constantes y variantes en el tiempo.

La principal fortaleza del sistema propuesto respecto a los sistemas encontrados en la literatura es su capacidad de operación simultánea de una técnica de MPPT y de la limitación de la derivada de corriente de carga de las baterías, para extender su vida útil. Adicionalmente, el artículo presenta un análisis detallado del sistema de control propuesto (i.e. análisis de transversalidad y alcanzabilidad del SMC y diseño del regulador $\mathrm{P}$ ) y el hardware requerido para la implementación del sistema de control es sencillo, lo cual facilita su implementación.

El resto del artículo está organizado como sigue: en la siguiente sección se presenta la estructura del cargador de baterías PV propuesto, posteriormente se presenta el modelo matemático y el diseño del convertidor utilizado. Luego, se describe el diseño del sistema de control y su implementación. Finalmente, se presentan los resultados de simulación y se cierra el artículo con las conclusiones.

\section{ESTRUCTURA DEL CARGADOR DE BATERÍAS FOTOVOLTAICO}

La Fig. 1 presenta la estructura propuesta para el cargador de baterías fotovoltaico. El sistema de potencia está formado por un convertidor Buck, el cual transfiere la potencia extraída por el arreglo fotovoltaico a la batería. El convertidor se debe controlar para cumplir dos condiciones: primero, asegurar que la derivada de la corriente impuesta a la batería está por debajo del límite de seguridad; y segundo, asegurar la operación del arreglo fotovoltaico en el punto de máxima potencia.

Para limitar la derivada de corriente impuesta a la batería se propone implementar un controlador de corriente, el cual estará gobernado por un limitador de referencia que impondrá la restricción a la derivada. De esta forma, se asegura que la corriente de la batería siempre cumple las restricciones dinámicas de seguridad. 


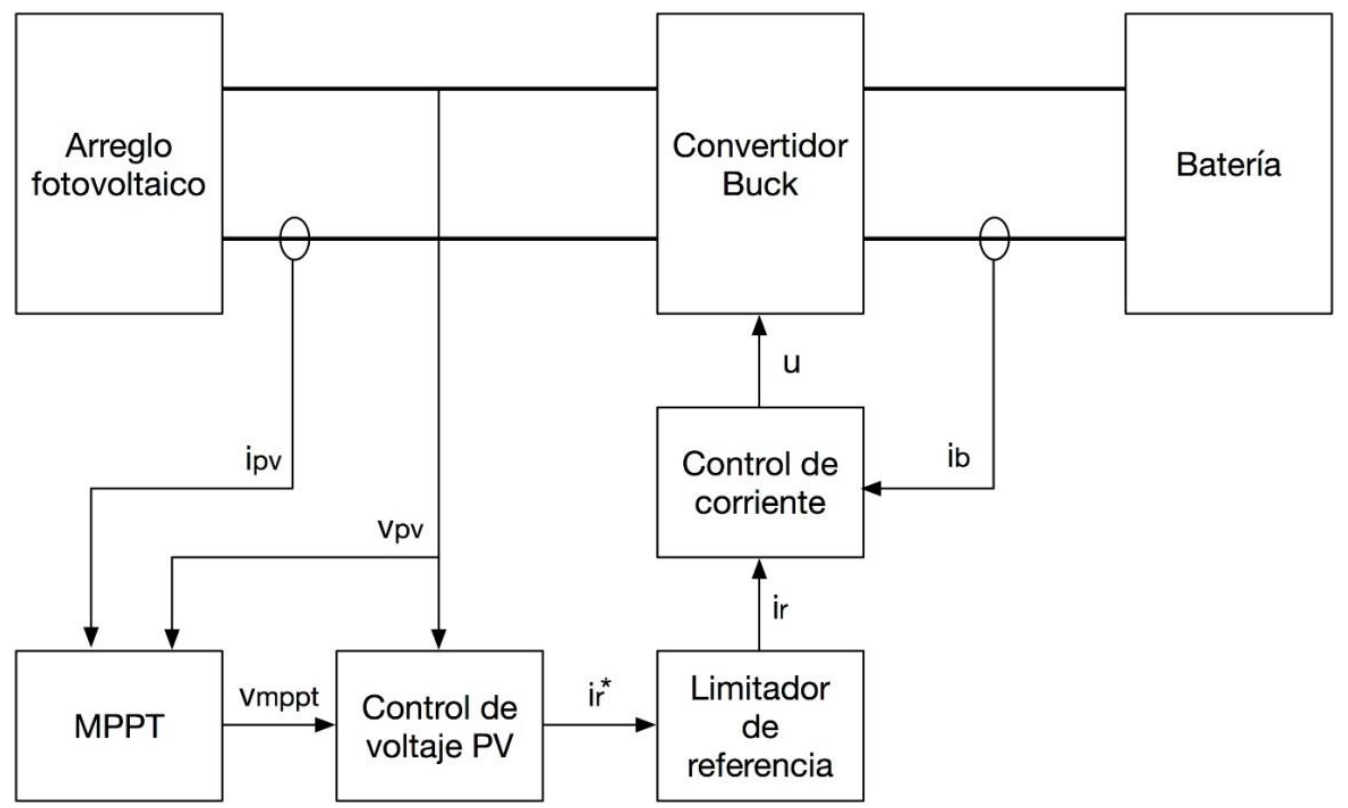

Fig. 1. Estructura del cargador de baterías fotovoltaico. Fuente: autores.

Para asegurar la máxima extracción de potencia al arreglo fotovoltaico (PV), se utiliza un algoritmo $\mathrm{P} \& \mathrm{O}$ para detectar el voltaje óptimo de operación del arreglo PV. Ese voltaje óptimo se impone, como referencia, a un controlador de voltaje, el cual a su vez impone la referencia al control de corriente. Esta estructura asegura que el convertidor buck impone al arreglo PV el voltaje óptimo, y al mismo tiempo, se limita la derivada de corriente a un valor seguro para la batería.

Es importante notar que el algoritmo P\&O perturba la referencia del voltaje PV en la dirección que incrementa la potencia producida por el arreglo PV, tal y como se describe en [27]. Por lo tanto, este algoritmo tiene un efecto integrador que compensa errores de estado estacionario en el controlador de voltaje, lo que permite reducir la complejidad de ese controlador. Esta característica permite simplificar la estructura de control para reducir costos de implementación. En las secciones siguientes se describe el diseño de cada una de las etapas del cargador de baterías PV.

\section{MODELO MATEMÁTICO Y DISEÑO DEL CONVERTIDOR}

La Fig. 2 presenta el esquema eléctrico del cargador de baterías PV, donde se observa el modelo no-lineal definido para representar el arreglo PV. Asimismo, en la figura se observa el convertidor Buck y el modelo clásico de batería.

El modelo del arreglo fotovoltaico, denominado modelo de diodo simple [28], está formado por una fuente de corriente $\left(i_{s c}\right)$ en paralelo con un diodo (elemento junto a la fuente de corriente en la Fig. 2). La fuente de corriente es proporcional a la irradiación solar y el diodo representa la no linealidad del arreglo PV. La ecuación que describe el modelo de diodo simple se reporta en (1) donde $i_{p v}$ y $v_{p v}$ son la corriente $\mathrm{y}$ voltaje del arreglo, respectivamente, el parámetro $A$ representa la corriente inversa de saturación del diodo y el parámetro $B$ depende de la temperatura. Una descripción detallada de estos parámetros se reporta en [28].

$i_{p v}=i_{S C}-A \cdot \exp \left(B \cdot v_{p v}\right)$ 


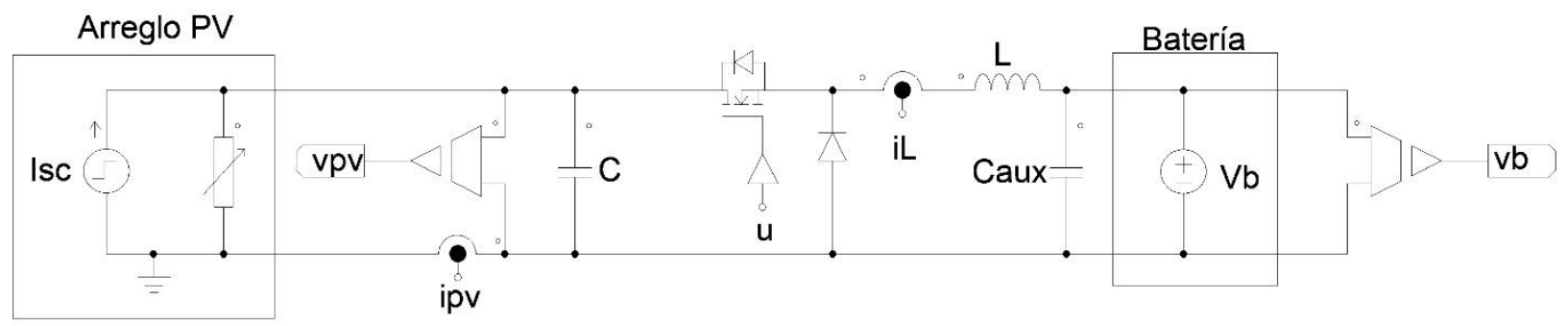

Fig. 2. Esquema eléctrico del cargador de beterías fotovoltaico. Fuente: autores.

La variable de control en este circuito corresponde a la señal binaria $u$, la cual impone el estado del MOSFET (y complementariamente del DIODO). En este circuito, las ecuaciones diferenciales que describen el comportamiento del voltaje PV $\left(v_{p v}\right)$ y de la corriente del inductor $\left(i_{L}\right)$ se reportan en (2) y (3), respectivamente, las cuales se obtienen a partir de los balances de carga y flujo aplicados al capacitor e inductor, respectivamente.

$\frac{d v_{p v}}{d t}=\frac{i_{p v}-i_{L} \cdot u}{C}$

$\frac{d i_{L}}{d t}=\frac{v_{p v} \cdot u-v_{b}}{L}$

Estas expresiones dependen del valor de la capacitancia $C$ y de la inductancia $L$. Asimismo, las ecuaciones diferenciales dependen de la corriente $\mathrm{PV}$, reportada en (1), y del voltaje de la batería $v_{b}$. En estado estacionario se tiene que las derivadas de las ecuaciones diferenciales son iguales a cero, por lo que (2) y (3) se pueden expresar como (4) y (5), respectivamente, donde $d$ (ciclo de trabajo) corresponde al valor promedio de $u$ :

$i_{p v}=i_{L} \cdot d$

$v_{b}=v_{p v} \cdot d$

Para proteger la batería del rizado de alta frecuencia generado por el convertidor de potencia, tradicionalmente se incluye un capacitor auxiliar $C_{a u x}$ con alta derivada de corriente entre el inductor y la batería, tal y como se presenta en la Fig. 2 de acuerdo con lo reportado en [8], [29]. Debido a que $C_{\text {aux }}$ absorbe el rizado de alta frecuencia, la corriente de la batería corresponde al valor promediado de la corriente del inductor dentro del periodo de conmutación, i.e. $i_{b}=$ $\left\langle i_{L}\right\rangle$. Es de notar que $C_{a u x}$ está conectado en paralelo con la batería, por tanto, su voltaje es igual a $v b$ y no introduce una nueva variable de estado en el circuito.

Teniendo en cuenta que uno de los objetivos del sistema de control es limitar la derivada de la corriente impuesta a la batería, la magnitud de la derivada de la corriente promedio del inductor debe ser menor a ese límite. Definiendo el límite de seguridad como $S R_{i}$, esta condición se formaliza así:

$\left|\frac{d i_{b}}{d t}\right|<S R_{i}$

$\left\langle i_{L}\right\rangle=i_{b}$

\section{CONTROL DE CORRIENTE}

Para controlar el comportamiento dinámico de la corriente en la batería se propone un SMC. Este tipo de controlador es una buena alternativa, ya que provee estabilidad global [19] y evita el uso de un circuito modulador [30], lo que simplifica el hardware requerido para su implementación y por lo tanto reduce su costo. Adicionalmente, el SMC de corriente contribuye a la reducción de las oscilaciones en la tensión del generador PV, contribuyendo al 
correcto seguimiento del punto de máxima potencia realizado por el MPPT.

Como se reporta en la Fig. 1, el SMC de corriente mide la corriente del inductor $i_{L} \mathrm{y}$ regula la activación del MOSFET y del DIODO para asegurar que $i_{L}$ sigue la referencia $i_{r}$, donde la corriente de la batería corresponde al valor promediado de la corriente del inductor dentro del periodo de conmutación, i.e. $i_{b}=\left\langle i_{L}\right\rangle$. Para implementar el sistema de control se define la función de conmutación $S_{x}$ y superficie de deslizamiento $S_{d}$ reportadas en (8).

$S_{x}=i_{L}-i_{r}, S_{d}=\left\{S_{x}=0\right\}$

Al reemplazar (3) en (8) se obtiene la derivada de la función de conmutación:

$\frac{d S_{x}}{d t}=\frac{d i_{L}}{d t}-\frac{d i_{r}}{d t}=\frac{v_{p v} \cdot u-v_{b}}{L}-\frac{d i_{r}}{d t}$

A partir de (9) se analizan las condiciones necesarias y suficientes para garantizar estabilidad y convergencia en un controlador por modos deslizantes [31]: condición de transversalidad y condición de alcanzabilidad, ya que en [31] se demostró que la condición de control equivalente se cumple en todo sistema con transversalidad y alcanzabilidad.

La condición de transversalidad se analiza a partir de (9) según lo reportado en (10):

$\frac{d}{d u}\left(\frac{d S_{x}}{d t}\right) \neq 0$

Reemplazando (9) en (10) se verifica el cumplimiento, y el signo, de la condición de transversalidad:

$\frac{d}{d u}\left(\frac{d S_{x}}{d t}\right)=\frac{v_{p v}}{L}>0$

En [32] se demostró que, con un signo positivo de transversalidad, las condiciones de alcanzabilidad son:

$$
\left.\lim _{S_{x} \rightarrow 0^{-}} \frac{d S_{x}}{d t}\right|_{u=1}>\left.0 \mathrm{y} \lim _{S_{x} \rightarrow 0^{+}} \frac{d S_{x}}{d t}\right|_{u=0}<0
$$

Reemplazando (9) en (12) se obtiene:

$$
\begin{aligned}
& \left.\lim _{S_{x} \rightarrow 0^{-}} \frac{d S_{x}}{d t}\right|_{u=1}=\frac{v_{p v}-v_{b}}{L}-\frac{d i_{r}}{d t}>0 \\
& \left.\lim _{S_{x} \rightarrow 0^{+}} \frac{d S_{x}}{d t}\right|_{u=0}=\frac{-v_{b}}{L}-\frac{d i_{r}}{d t}<0
\end{aligned}
$$

Teniendo en cuenta que $v_{p v}>v_{b}$, las expresiones (13) y (14) verifican las condiciones de alcanzabilidad de la superficie, asegurando la estabilidad global del SMC, siempre que la señal de referencia $i_{r}$ cumpla con la restricción dinámica reportada en (15). Esta restricción se discute en la sección dedicada a la implementación del controlador.

$\frac{-v_{b}}{L}<\frac{d i_{r}}{d t}<\frac{v_{p v}-v_{b}}{L}$

Finalmente, la existencia del modo de deslizamiento garantiza la operación del sistema dentro de la superficie de deslizamiento con una trayectoria paralela a la misma como se reporta en (16) [19], [20], [32].

$\left\{S_{x}=0\right\},\left\{\frac{d S_{x}}{d t}=0\right\}$

Bajo esas condiciones de estabilidad, la dinámica equivalente en lazo cerrado depende de la función de deslizamiento (8) y de la ecuación diferencial restante, i.e. (2), así:

$i_{L}=i_{r}, \frac{d v_{p v}}{d t}=\frac{i_{p v}-i_{r} \cdot d}{C}$

La expresión (17) se utilizará en la siguiente sección para diseñar el controlador de voltaje. 


\section{CONTROL DE VOLTAJE}

A partir de la dinámica equivalente del SMC, i.e. expresión (17), se obtienen dos funciones de transferencia para describir el comportamiento del voltaje PV $v_{p v}$ : (18) reporta el cambio de $v_{p v}$ dependiendo de $i_{r}$, y (19) reporta el cambio de $v_{p v}$ dependiendo de $i_{p v}$.

$\frac{v_{p v}(s)}{i_{r}(s)}=\frac{-d}{C \cdot s}$

$\frac{v_{p v}(s)}{i_{p v}(s)}=\frac{1}{C \cdot s}$

El control de voltaje se implementa en cascada, según lo anticipado en la Fig. 1, utilizando la estructura de lazo cerrado reportada en la Fig. 3. Ese diagrama de bloques pone en evidencia el intercambio de señales entre las funciones de transferencia (18) y (19), la variable a controlar $v_{p v}$ y las señales de referencia $v_{r} \mathrm{y}$ de perturbación $i_{p v}$. Es importante recordar que $v_{r}$ se genera con un algoritmo $\mathrm{P} \& \mathrm{O}$, el cual tiene una acción tipo integral. Esta condición permite implementar el controlador de voltaje $G_{v}(s)$ utilizando una ganancia proporcional, lo que simplifica la implementación de la estructura de control.

Asumiendo $G_{v}(s)=k_{p}$, es decir un controlador proporcional con ganancia negati- va, la función de transferencia de lazo cerrado entre $v_{r}$ y $v_{p v}(20)$ :

$\frac{v_{p v}(s)}{v_{r}(s)}=\frac{1}{\left(\frac{C}{d \cdot k_{p}}\right) \cdot s+1}$

De forma similar, la función de transferencia de lazo cerrado entre $i_{p v}$ y $v_{p v}(21)$ :

$\frac{v_{p v}(s)}{i_{p v}(s)}=\frac{\left(\frac{1}{d \cdot k_{p}}\right)}{\left(\frac{C}{d \cdot k_{p}}\right) \cdot s+1}$

La dinámica reportada en (20) demuestra que el error de estado estacionario entre $v_{r} \mathrm{y} v_{p v}$ es cero. Pero, la dinámica reportada en (21) demuestra que cualquier valor en $i_{p v}$ diferente de cero, se multiplica por $\frac{1}{d \cdot k_{p}}$ para producir un error de estado estacionario. Es decir, entre mayor sea el valor de $k_{p}$ menor será el error de estado estacionario. En todo caso, la acción integrar del algoritmo P\&O corregirá el error de estado estacionario.

A partir de (20) y (21) se observa que la constante de tiempo equivalente de $v_{p v}$, en lazo cerrado, (22):

$\tau=\frac{C}{d \cdot k_{p}}$

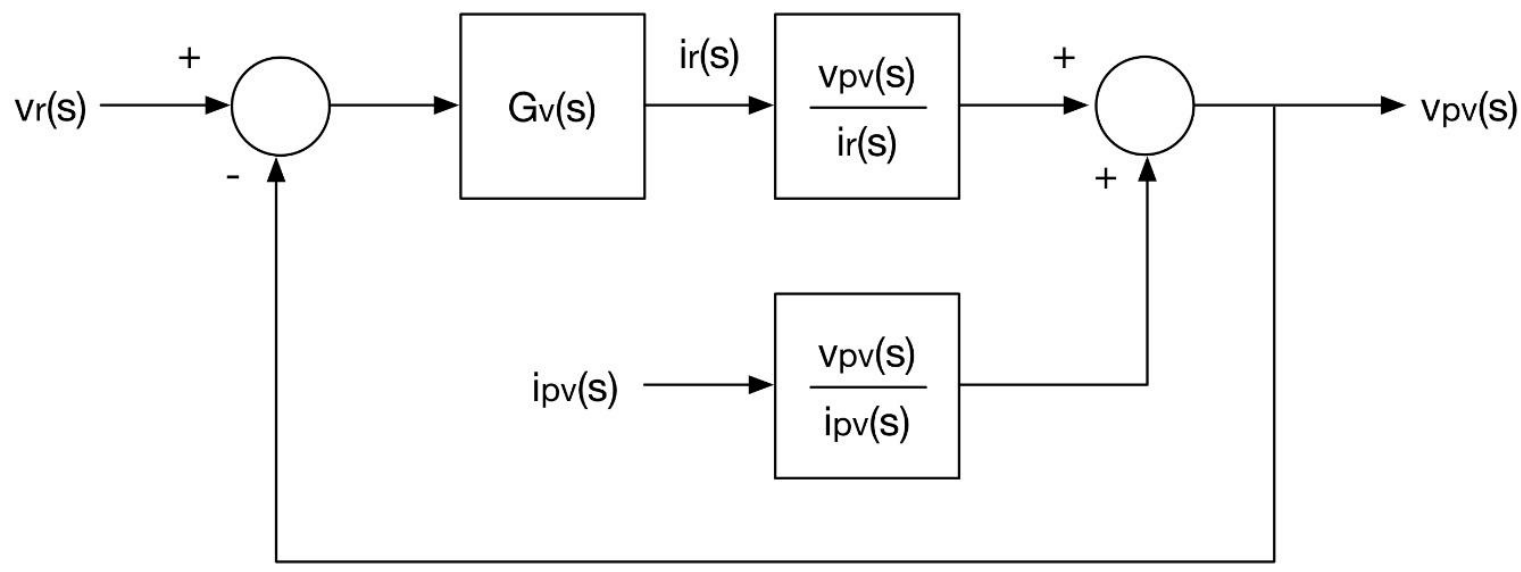

Fig. 3. Control de voltaje en cascada. Fuente: autores. 
Considerando que el tiempo de estabilización de un sistema de primer orden es aproximadamente $t_{s}=4 \cdot \tau$ [19], [33], la expresión se reescribe según (23) para permitir el diseño de $k_{p}$ a partir del tiempo de estabilización deseado para $v_{p v}$, el cual depende del tiempo de perturbación $T_{a}$ del P\&O, según lo reportado en (24), para garantizar estabilidad [19], [27].

$k_{p}=-\frac{4 \cdot C}{d \cdot t_{s}}$

$T_{a}>t_{s}$

\section{IMPLEMENTACIÓN DEL SISTEMA DE CONTROL}

El controlador de corriente se debe implementar siguiendo la ley de control definida en (13) y (14), es decir, si $S_{x}<0$ se impone $u=1$, si $S_{x}>0$ se impone $u=0$. Asimismo, se incluye una banda de histéresis para limitar la frecuencia de conmutación según lo reportado en [19], [20].

La Fig. 4 presenta el circuito diseñado para implementar los sistemas de control de corriente y voltaje. Inicialmente, se implementa un comparador con histéresis basado en dos comparadores y un Flip-Flop S-R, el cual impone la ley de control. Ese comparador por histéresis procesa la señal $S_{x}$ calculada a partir de $i_{L}$ e $i_{r}$.

Para limitar la derivada de la corriente de la batería $i_{b}$, es decir la corriente promedio de $i_{L}$, se introduce un circuito limitador de derivada [34] entre el controlador voltaje $G v(s)=k_{p}$ y la referencia del SMC, tal y como se ilustra en la Fig. 4. Por tanto, la señal de referencia del SMC $i_{r}(25)$

$i_{r}=\left\{\begin{array}{ccc}i_{r}^{*} & \text { si } & \left|\frac{d i_{r}^{*}}{d t}\right|<S R_{i} \\ \int S R_{i} d t & \text { si } & \left|\frac{d i_{r}^{*}}{d t}\right|>S R_{i}\end{array}\right.$
Donde $i_{r}{ }^{*}$ es referencia generada por el controlador proporcional y $\left|\frac{d i_{r}^{*}}{d t}\right|$ es la magnitud de la derivada de $i_{r}{ }^{*}$. La expresión (25) impone una señal de referencia al SMC igual a la definida por $k_{p}$ si $\left|\frac{d i_{r}^{*}}{d t}\right|<$ $S R_{i}$; de lo contrario, si $\left|\frac{d i_{r}^{*}}{d t}\right|>S R_{i}$, la señal de referencia del SMC será, en el peor caso, una rampa con derivada igual a $S R_{i}$. De esta forma se garantiza el cumplimiento del límite de seguridad definido en (6), lo que impone una derivada de corriente en la batería dentro de los límites de seguridad para evitar reducir su vida útil.

Por otra parte, para garantizar la estabilidad del SMC de corriente, la restricción dinámica impuesta en (15) debe cumplirse para toda condición de operación. Esto se implementa utilizando el limitador de derivada para $i_{r}$ previamente descrito, sincronizando los límites de (6) y (15):

$\max \left|\frac{-v_{b}}{L}\right|<S R_{i}$
$\max \left|\frac{v_{p v}-v_{b}}{L}\right|<S R_{i}$

Las expresiones (26) y (27) se utilizan para diseñar el inductor a partir de los voltajes máximos del arreglo $\mathrm{PV}$, de la batería y el límite seguro para la derivada de la corriente. Teniendo en cuenta que $v_{b}$ $>0$ y $v_{p v}>v b$, el diseño del inductor se debe realizar según (28) para garantiza el cumplimiento de la restricción dinámica (15), asegurando así la estabilidad del SMC.

$\frac{\max \left(v_{b}\right)}{S R_{i}}<L$ y $\frac{\max \left(v_{p v}-v_{b}\right)}{S R_{i}}<L$

La implementación de la banda de histéresis $(H)$ del SMC se realiza con dos fuentes de tensión de $+H / 2 \mathrm{~V} \mathrm{y}-\mathrm{H} / 2 \mathrm{~V}$, dos sumadores y dos comparadores cuyas salidas se conectan a los terminales Set y Reset del Flip-Flop, como se describe en la Fig. 4. 
Cargador de baterías fotovoltaico con control por modos deslizantes y limitación de la derivada de corriente de carga

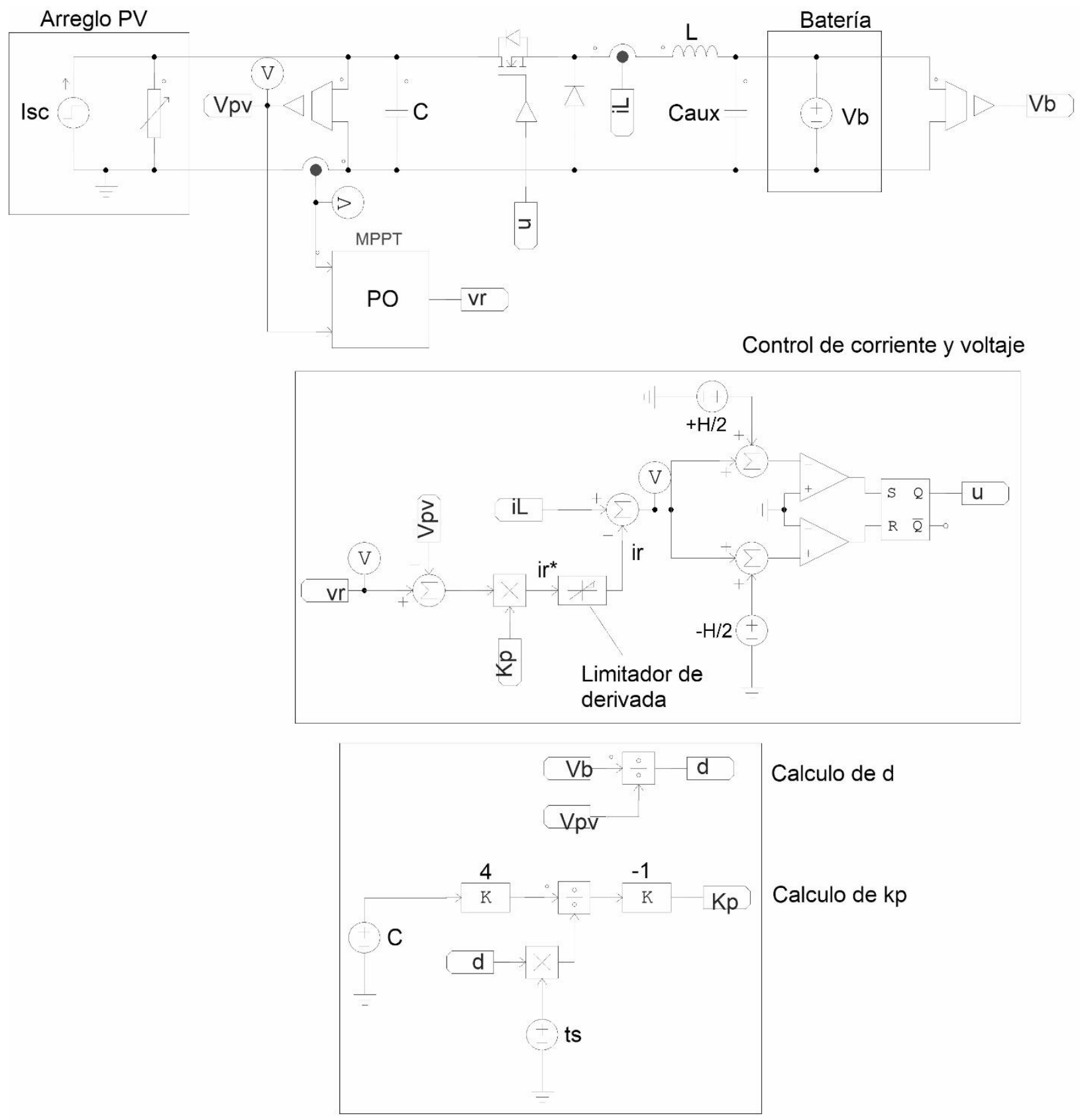

Fig. 4. Cargador de baterías fotovoltaico con sistema de control. Fuente: autores.

El controlador de voltaje se implementa con la ganancia $k_{p}$ calculada según lo reportado en (23), lo que requiere a su vez el cálculo continuo del ciclo de trabajo usando la relación reportada en (5). Finalmente, la referencia del controlador de voltaje la genera el algoritmo $\mathrm{P} \& \mathrm{O}$ para maximizar la potencia producida por el arreglo fotovoltaico.

\section{SIMULACIONES CIRCUITALES}

Para ilustrar el desempeño del sistema de control, el circuito de la Fig. 4 se parametriza con los siguientes valores: $L=100$ $\mu H, C=120 \mu F, v_{b}=6 \mathrm{~V}$. Este ejemplo considera un módulo BP585 con los siguientes parámetros: $A=8,95 e-7 A$ y $B=$ $1,406 V^{-1}$. El algoritmo $\mathrm{P} \& \mathrm{O}$ se diseñó se- 
gún lo reportado en [27], con un tiempo de perturbación $T_{a}=0,5 \mathrm{~ms}$ y una amplitud de perturbación de $250 \mathrm{mV}$. Para garantizar la estabilidad del $\mathrm{P} \& \mathrm{O}$, siguiendo la restricción (24), el tiempo de estabilización del voltaje PV se impuso igual a $t_{s}=0,25$ $m s$. Finalmente, se asume que la batería soporta una derivada máxima de corriente $S R_{i}=0,005 \mathrm{~A} / \mu \mathrm{s}$, tomando como referencia sistemas de prueba de baterías que ofrecen derivadas de corriente similares para crear perfiles de carga y descarga [35], [36]. Sin embargo, $\mathrm{SR}_{\mathrm{i}}$ se puede definir de acuerdo con la aplicación particular y a las características de la batería a utilizar. A partir de los datos anteriores, la banda de histéresis del comparador se define igual a 0,2 A, i.e. $H=0,2 \mathrm{~V}$ en el circuito de la Fig. 4, para proveer una frecuencia de conmutación nominal de $100 \mathrm{kHz}$. Finalmente, los valores seleccionados para $L$ y $S R_{i}$ cumplen con la restricción (28), por tanto, se cumple la restricción dinámica (15) que garantiza la estabilidad global del SMC.

Para evaluar el desempeño del sistema de control se considera el arranque del sistema, desde condiciones iniciales nulas, a una irradiación solar de $1000 \mathrm{~W} / \mathrm{m}^{2}$. La Fig. 5 presenta los resultados de la simulación en PSIM, donde se observa que el voltaje PV llega a un régimen estable de tres puntos y la potencia producida es igual a $42,5 \mathrm{~W}$, lo que corresponde a la máxima potencia posible con una irradiación solar de $1000 \mathrm{~W} / \mathrm{m}^{2}$.

La Fig. 6 presenta un zoom alrededor de $2 \mathrm{~ms}$, donde se observa que la referencia de corriente $i_{r}$ (señal azul) impone la limitación de derivada requerida. Asimismo, la corriente del inductor sigue adecuadamente la referencia, lo que impone la restricción dinámica a la corriente de la batería, protegiendo de esta forma el almacenador de energía.
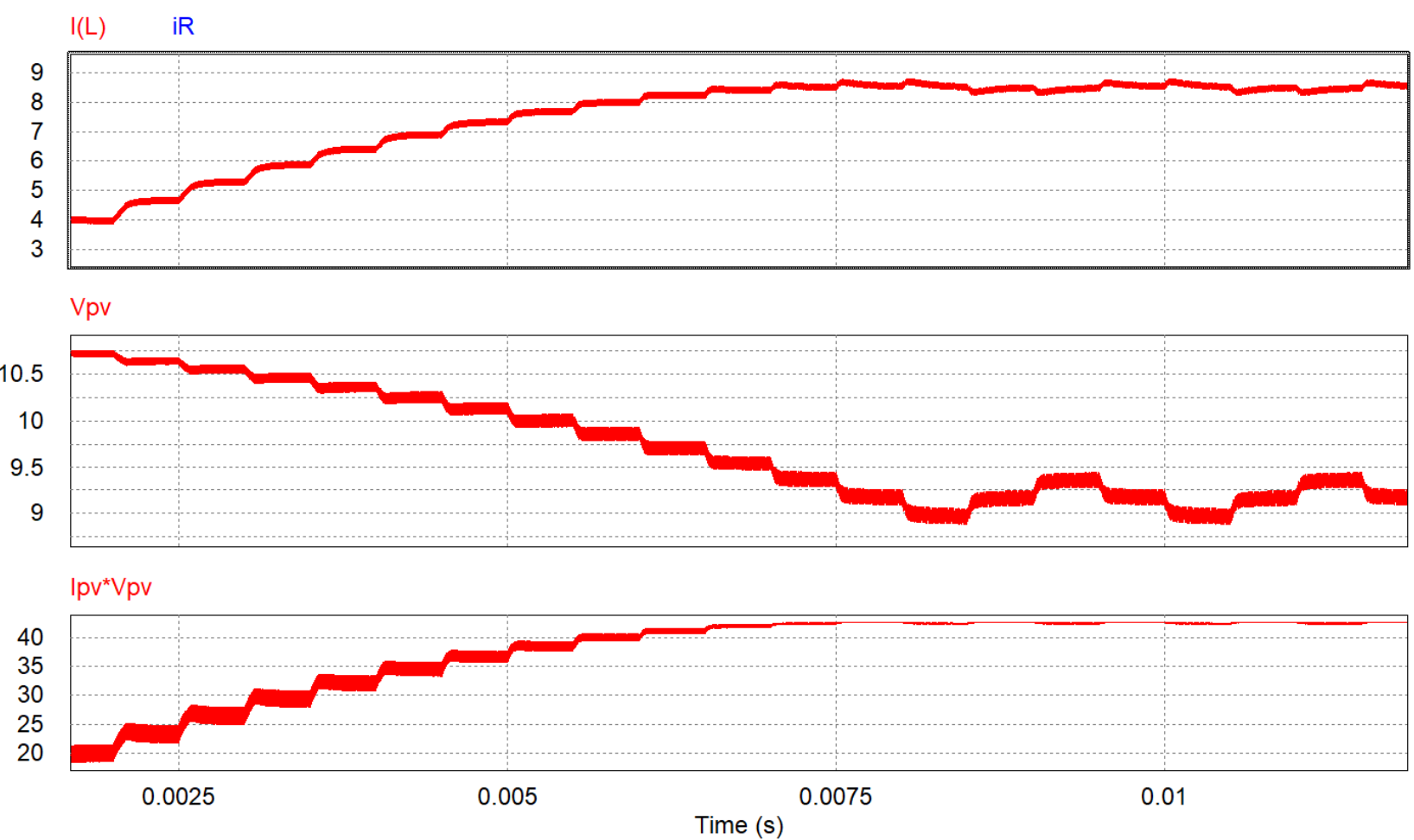

Fig. 5. Simulación de seguimiento del punto de máxima potencia. Unidades: corriente en el inductor $(I(L))$ y su referencia $(i R)$ en $A$, tensión del panel ( $V p v)$ en $V$ y potencia del panel $\left(I p v^{*} V p v\right)$ en $W$. Fuente: autores. 
Cargador de baterías fotovoltaico con control por modos deslizantes y limitación de la derivada de corriente de carga

$\mathrm{I}(\mathrm{L}) \quad \mathrm{iR}$

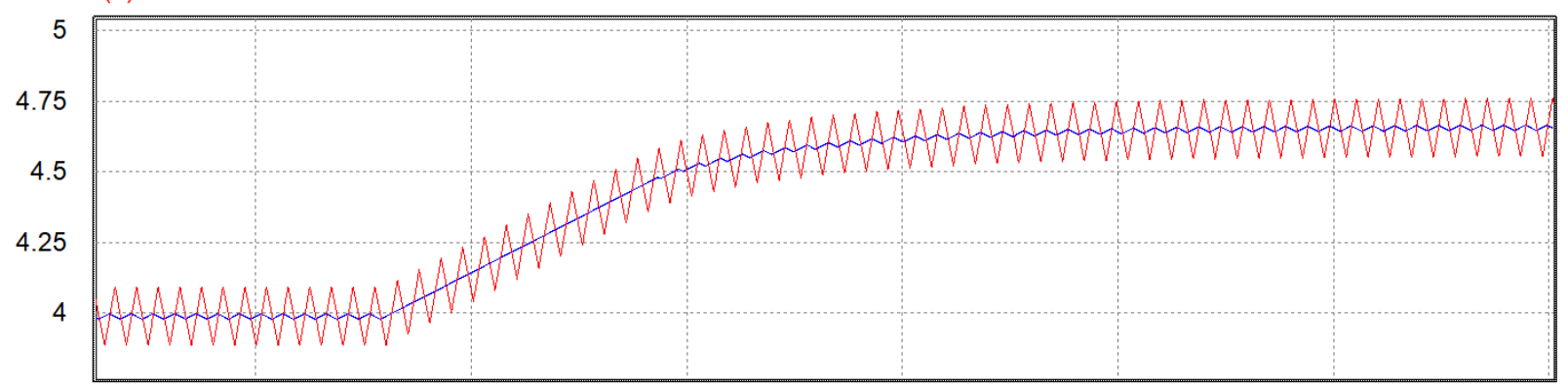

Sx

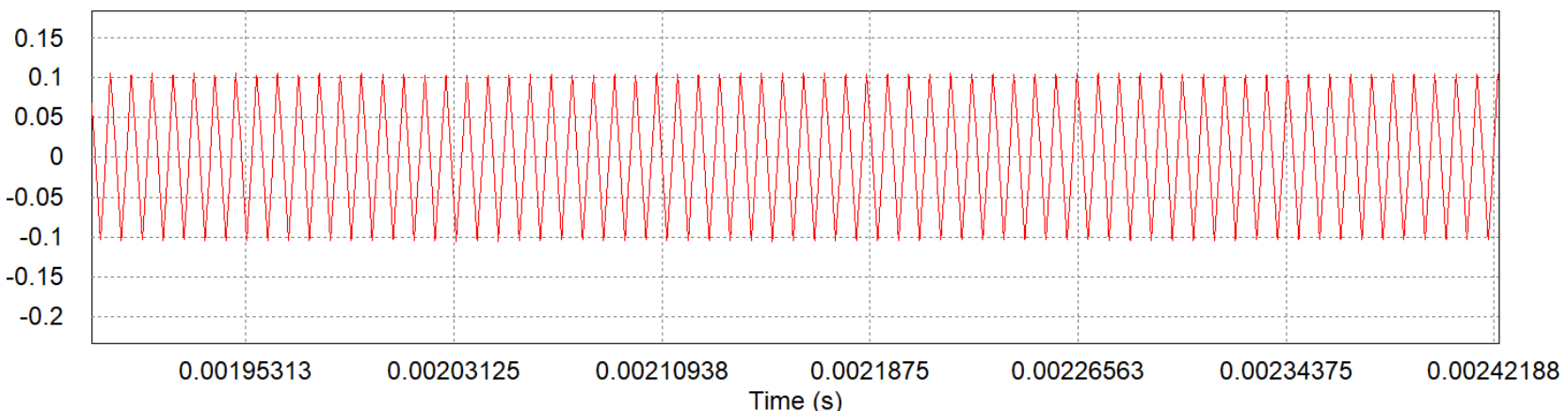

Fig. 6. Zoom a Fig. 5. Unidades: corriente en el inductor $(I(L))$ y su referencia $(i R)$ en $A$ y función de conmutación $(S x)$ en $A$. Fuente: autores.
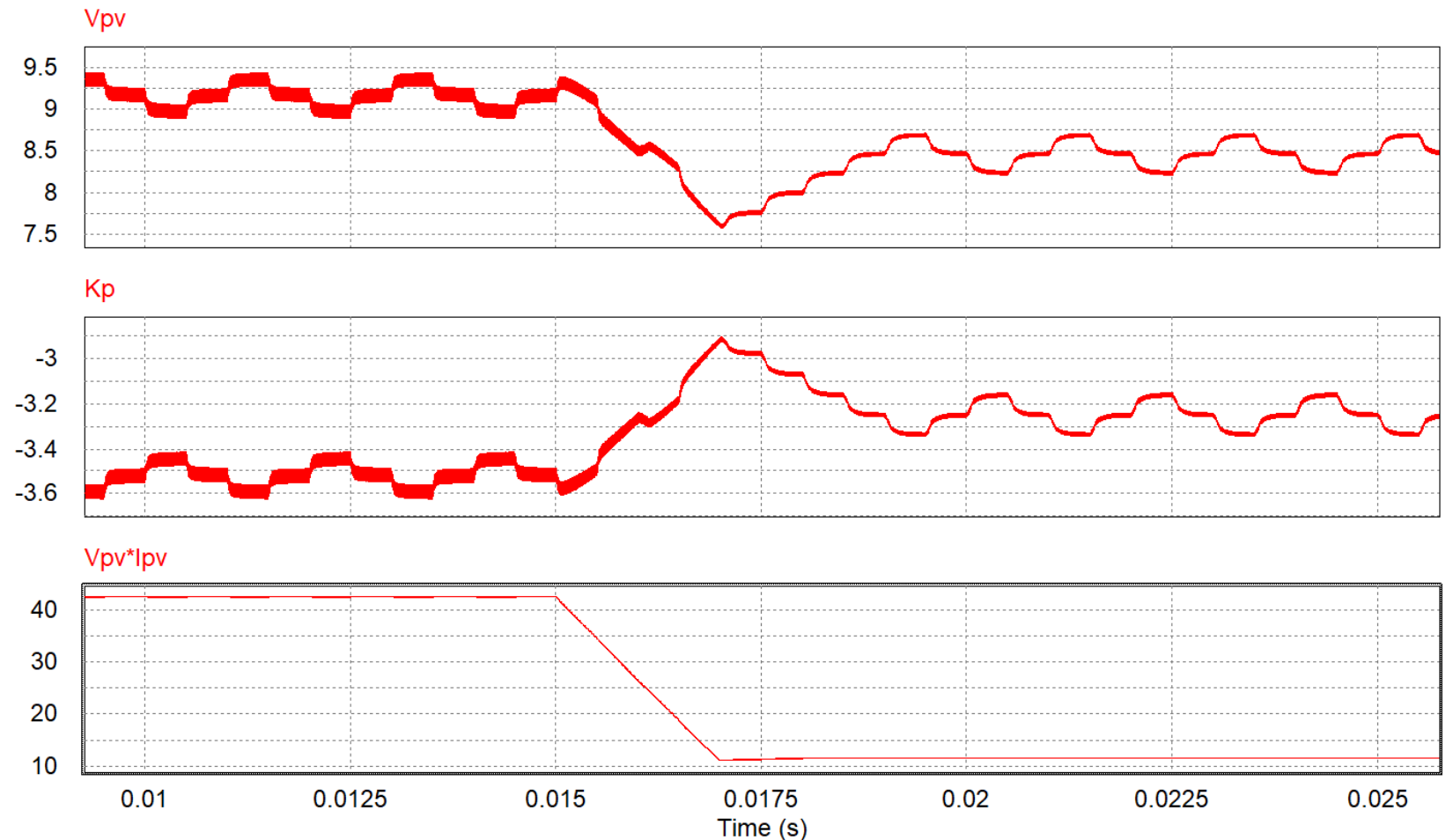

Fig. 7. Condición dinámica de irradiación solar. Unidades: tensión del panel $(V p v)$ en $V$, constante del controlador de tensión $(K p)$ es $F / s$, y potencia del panel $\left(I p v^{*} V p v\right)$ en $W$. Fuente: autores. 
La Fig. 7 presenta el desempeño del sistema de control ante un cambio rápido en la irradiación solar: en este caso, la irradiación cambia de $1000 \mathrm{~W} / \mathrm{m}^{2}$ a $300 \mathrm{~W} / \mathrm{m}^{2}$ en un intervalo de tiempo de $2 \mathrm{~ms}$. La simulación confirma que el sistema de control responde adecuadamente, ya que el voltaje del panel llega nuevamente a un régimen estable de tres puntos, lo que garantiza alcanzar el punto de máxima potencia. Asimismo, la figura reporta el cálculo dinámico de $k_{p}$ para proveer el tiempo de estabilización deseado, de forma que se garantiza la estabilidad del algoritmo P\&O, i.e se cumple (24).

Finalmente, las simulaciones presentadas en esta sección demuestran el funcionamiento esperado del sistema: se limita la derivada de la corriente de la batería, y al mismo tiempo, se realiza un seguimiento del punto de trabajo que maximiza la producción de energía. Asimismo, las simulaciones demuestran que la inclusión del limitador de derivada no afecta la estabilidad de los controladores de corriente y voltaje.

\section{CONCLUSIONES}

En este artículo se presentó un sistema cargador de baterías fotovoltaico capaz de implementar, simultáneamente, una técnica de MPPT (P\&O) y de limitar la derivada de corriente de carga de las baterías. El sistema se implementó con un convertidor Buck y un sistema de control compuesto por un P\&O y un control en cascada. El $\mathrm{P} \& O$ genera la tensión de referencia del panel $\left(v_{r}\right)$ para seguir el MPP. El lazo externo del control en cascada toma dicha referencia y calcula la referencia de la corriente del inductor $\left(i_{r}{ }^{*}\right)$ como acción de control, la cual pasa por un limitador de derivada para obtener la referencia efectiva $\left(i_{r}\right)$ del lazo interno de control de corriente. Finalmente, $i_{b}$ es indirectamente regulada por el SMC de corriente que ga- rantiza la estabilidad del convertidor en cualquier condición de operación.

El sistema propuesto se implementó en PSIM para validar su funcionalidad y verificar su desempeño. Las simulaciones muestran que el sistema propuesto realiza correctamente el seguimiento del punto de máxima potencia ante variaciones de la irradiancia mientras limita la derivada de corriente de carga de las baterías. Las simulaciones también muestran que la implementación del sistema de control es simple y se puede realizar en un microcontrolador de bajo costo o incluso de forma análoga.

Actualmente, los autores están trabajando en el desarrollo de un sistema de control de más alto nivel que incluya una estrategia de gestión de carga de la batería, e.g. carga por corriente constantetensión constante. Además, dicho algoritmo podría incluir condiciones de desconexión de la batería, del panel y/o de la carga en ciertas condiciones de operación. Sin embargo, dicha estrategia utilizaría el sistema de control propuesto ( $\mathrm{P} \& \mathrm{O}$ y control en cascada) como el sistema de control de bajo nivel para garantizar la estabilidad del sistema.

\section{AGRADECIMIENTOS}

Este trabajo fue financiado por la Universidad Nacional de Colombia y la Universidad Industrial de Santander, bajo los proyectos GESMICRO-30773 y Capital Semilla-1896, respectivamente. El trabajo también fue financiado por el proyecto UNAL-ITM-39823/P17211, el cual es desarrollado por la Universidad Nacional de Colombia y el Instituto Tecnológico Metropolitano.

\section{REFERENCIAS}

[1] REN21, "Advancing the global renewable energy transition," 2017.

[2] IEA-PVPS, "2016 Snapshot of Global 
Cargador de baterías fotovoltaico con control por modos deslizantes y limitación de la derivada de corriente de carga

Photovoltaic Markets," 2017.

[3] IDEAM, "Atlas de radiación solar, ultravioleta y ozono de Colombia," Atlas, 2014. [Online]. Available: http://atlas.ideam.gov.co/visorAtlasRadiacion .html.

[4] World Bank Group, "Global Solar Atlas," 2016. [Online]. Available: https://olc.worldbank.org/content/globalsolar-atlas.

[5] F. Palmiro, R. Rayudu, and R. Ford, "Modelling and simulation of a solar PV lithium ion battery charger for energy kiosks application," in 2015 IEEE PES Asia-Pacific Power and Energy Engineering Conference (APPEEC), 2015, vol. 3, pp. 1-5.

[6] R. A. Messenger and J. Ventre, Photovoltaics Systems Engineering, Second. Boca Raton, London, New York, Washigton D.C.: Taylor \& Francis, 2004.

[7] Y. E. Abu Eldahab, N. H. Saad, and A. Zekry, "Enhancing the design of battery charging controllers for photovoltaic systems," Renew. Sustain. Energy Rev., vol. 58, pp. 646-655, May 2016.

[8] J. López, S. I. Seleme, P. F. Donoso, L. M. F. Morais, P. C. Cortizo, and M. A. Severo, "Digital control strategy for a buck converter operating as a battery charger for standalone photovoltaic systems," Sol. Energy, vol. 140, pp. 171-187, Dec. 2016.

[9] A. M. Gee, F. V. P. Robinson, and R. W. Dunn, "Analysis of Battery Lifetime Extension in a Small-Scale Wind-Energy System Using Supercapacitors," IEEE Trans. Energy Convers., vol. 28, no. 1, pp. 24-33, Mar. 2013.

[10] J. Li, A. M. Gee, M. Zhang, and W. Yuan, "Analysis of battery lifetime extension in a SMES-battery hybrid energy storage system using a novel battery lifetime model," Energy, vol. 86, pp. 175-185, Jun. 2015.

[11] G. Ning, B. Haran, and B. N. Popov, "Capacity fade study of lithium-ion batteries cycled at high discharge rates," J. Power Sources, vol. 117, no. 1-2, pp. 160-169, May 2003.

[12] J. Li, R. Xiong, Q. Yang, F. Liang, M. Zhang, and W. Yuan, "Design/test of a hybrid energy storage system for primary frequency control using a dynamic droop method in an isolated microgrid power system," Appl. Energy, vol. 201, pp. 257-269, Sep. 2017.

[13] J. Li, Q. Yang, F. Robinson, F. Liang, M. Zhang, and W. Yuan, "Design and test of a new droop control algorithm for a SMES/battery hybrid energy storage system," Energy, vol. 118, pp. 1110-1122, Jan. 2017.

[14] Sony Energy Devices Corporation and Device
Solutions Business Group, "Lithium Ion Rechargeable Battery Technical Information," 2012.

[15] E. A. Jiménez-Brea, E. I. Ortiz-Rivera, A. Salazar-Llinás, and J. González-Llorente, "Simple photovoltaic solar cell dynamic sliding mode controlled maximum power point tracker for battery charging applications," in Conference Proceedings IEEE Applied Power Electronics Conference and Exposition - APEC, 2010, pp. 666-671.

[16] A. M. S. S. Andrade, E. Mattos, C. O. Gamba, L. Schuch, and M. L. da S. Martins, "Design and implementation of PV power zeta converters for battery charger applications," in 2015 IEEE Energy Conversion Congress and Exposition (ECCE), 2015, pp. 31353142.

[17] A. M. S. S. Andrade, L. Schuch, and M. L. da S. Martins, "Photovoltaic battery charger based on the Zeta converter: Analysis, design and experimental results," in 2015 IEEE 24th International Symposium on Industrial Electronics (ISIE), 2015, pp. 379-384.

[18] A. M. S. S. Andrade, R. C. Beltrame, L. Schuch, and M. L. da S. Martins, "PV module-integrated single-switch DC/DC converter for PV energy harvest with battery charge capability," in 2014 11th IEEE/IAS International Conference on Industry Applications, 2014, vol. 1, pp. 1-8.

[19] D. G. Montoya, C. A. Ramos-Paja, and R. Giral, "Improved Design of Sliding-Mode Controllers Based on the Requirements of MPPT Techniques," IEEE Trans. Power Electron., vol. 31, no. 1, pp. 235-247, Jan. 2016.

[20] D. González-Montoya, C. A. Ramos-Paja, and R. Giral, "Maximum power point tracking of photovoltaic systems based on the sliding mode control of the module admittance," Electr. Power Syst. Res., vol. 136, pp. 125134, Jul. 2016.

[21] R. Haroun, A. El Aroudi, A. Cid-Pastor, G. Garica, C. Olalla, and L. Martinez-Salamero, "Impedance Matching in Photovoltaic Systems Using Cascaded Boost Converters and Sliding-Mode Control," IEEE Trans. Power Electron., vol. 30, no. 6, pp. 31853199, Jun. 2015.

[22] A. Cid-Pastor, L. Martínez-Salamero, A. El Aroudi, R. Giral, J. Calvente, and R. Leyva, "Synthesis of loss-free resistors based on sliding-mode control and its applications in power processing," Control Eng. Pract., vol. 21, no. 5, pp. 689-699, May 2013.

[23] J. Guacaneme, D. González, and C. Trujillo, "Controlador difuso inteligente para un cargador de baterías de plomo-ácido," Ingeniería, vol. 8, no. 2, pp. 62-67, 2003. 
Cargador de baterías fotovoltaico con control por modos deslizantes y limitación de la derivada de corriente de carga

[24] SMA, "Technical Information Battery Management of the Sunny Island,” 2017.

[25] O. López-Santos, D. A. Zambrano Prada, Y. A. Aldana-Rodríguez, H. A. Esquivel-Cabeza, G. García, and L. Martínez-Salamero, "Control of a Bidirectional Cûk Converter Providing Charge/Discharge of a Battery Array Integrated in DC Buses of Microgrids," in Applied Computer Sciences in Engineering, J. C. Figueroa-García, E. R. López-Santana, J. L. Villa-Ramírez, and R. Ferro-Escobar, Eds. Cham: Springer International Publishing, 2017, pp. 495-507.

[26] M. E. Sahin, H. I. Okumus, and H. Kahveci, "Sliding mode control of PV powered DC/DC Buck-Boost converter with digital signal processor," in 2015 17th European Conference on Power Electronics and Applications (EPE'15 ECCE-Europe), 2015, pp. 1-8.

[27] N. Femia, G. Petrone, G. Spagnuolo, and M. Vitelli, "Optimization of Perturb and Observe Maximum Power Point Tracking Method," IEEE Trans. Power Electron., vol. 20, no. 4, pp. 963-973, Jul. 2005.

[28] G. Petrone and C. A. Ramos-Paja, "Modeling of photovoltaic fields in mismatched conditions for energy yield evaluations," Electr. Power Syst. Res., vol. 81, no. 4, pp. 1003-1013, Apr. 2011.

[29] Y.-C. Chuang and Y.-L. Ke, "Analysis and implementation of zero voltage switching integrated buck-flyback converter," IET
Power Electron., vol. 6, no. 7, pp. 2846-2852, 2011.

[30] Siew-Chong Tan, Y. M. Lai, and C. K. Tse, "General Design Issues of Sliding-Mode Controllers in DC-DC Converters," IEEE Trans. Ind. Electron., vol. 55, no. 3, pp. 1160-1174, Mar. 2008.

[31] H. Sira-Ramírez, "Sliding Motions in Bilinear Switched Networks," IEEE Trans. Circuits Syst., vol. 34, no. 8, pp. 919-933, 1987.

[32] P. A. Ortiz-Valencia and C. A. Ramos-Paja, "Sliding-mode controller for maximum power point tracking in grid-connected photovoltaic systems," Energies, vol. 8, no. 11, pp. 1236312387, 2015.

[33] C. A. Ramos-Paja, D. González, and A. J. Saavedra-Montes, "Accurate calculation of settling time in second order systems: A photovoltaic application," Rev. Fac. Ing. Univ. Antioquia, no. 66, pp. 104-117, 2013.

[34] T. Green, "TI Precision Designs: Reference Design - Single Op-Amp Slew Rate Limiter," Texas Instruments Incorporated, 2013.

[35] Digatron, "Universal Battery Tester ME Series," Digatron. [Online]. Available: http://www.digatron.com/zh/automotivebattery/universal-battery-tester/.

[36] Bitrode, "MCV-EV/HEV Battery Cell Testter," 2015. [Online]. Available: http://www.bitrode.com/model-mcv/. 